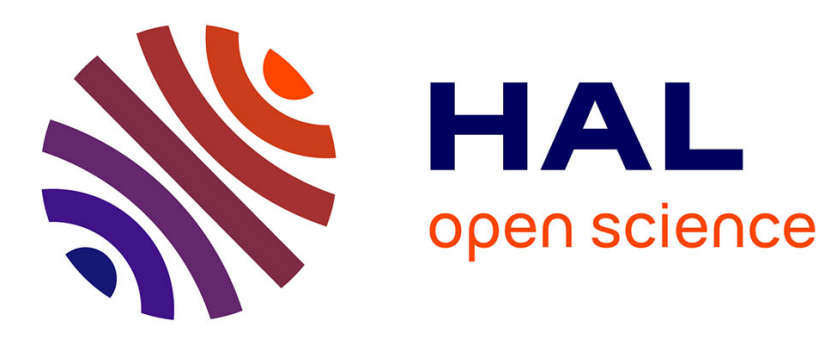

\title{
Microglia Reactivity: Heterogeneous Pathological Phenotypes
}

Hélène Hirbec, François Rassendren, Etienne Audinat

\section{To cite this version:}

Hélène Hirbec, François Rassendren, Etienne Audinat. Microglia Reactivity: Heterogeneous Pathological Phenotypes. Microglia Methods and Protocols, pp.41-55, 2019, 10.1007/978-1-4939-9658-2_4 . hal-02335930

\section{HAL Id: hal-02335930 \\ https://hal.science/hal-02335930}

Submitted on 19 Nov 2020

HAL is a multi-disciplinary open access archive for the deposit and dissemination of scientific research documents, whether they are published or not. The documents may come from teaching and research institutions in France or abroad, or from public or private research centers.
L'archive ouverte pluridisciplinaire HAL, est destinée au dépôt et à la diffusion de documents scientifiques de niveau recherche, publiés ou non, émanant des établissements d'enseignement et de recherche français ou étrangers, des laboratoires publics ou privés. 


\section{Microglia reactivity: Heterogeneous pathological phenotypes}

Hélène Hirbec, François Rassendren, Etienne Audinat

IGF, CNRS, INSERM, University of Montpellier, Montpellier, France

Running Head: Microglia reactive states

\section{Corresponding authors:}

Hélène Hirbec \& Etienne Audinat

Institute of Functional Genomics

Department of Neuroscience,

CNRS UMR 5203 - INSERM U1191, University of Montpellier,

141 rue de la Cardonille,

34094 Montpellier, France.

Emails : helene.hirbec@igf.cnrs.fr ; etienne.audinat@igf.cnrs.fr 


\begin{abstract}
A century ago, Pío del Río-Hortega discovered that microglial cells are endowed with remarkable dynamic and plastic capabilities. The real time plasticity of microglia could be revealed, however, only during the last 15 years with the development of new transgenic animal models and new molecular and functional analysis methods. Phenotyping microglia in situ with these new tools sealed the fate of the classical two state model of "resting" microglia in physiological conditions and "activated" microglia in pathological conditions. Our current view on functional behavior of microglia takes into account the exquisite reactivity of these immune cells to changes occurring in the CNS in both physiological and pathological conditions. We briefly review here the results and methods that have uncovered the dynamics and versatility of microglial reactivity.
\end{abstract}

Key words: microglial reactivity, microglial activation, brain macrophage, immune response, inflammation, neurodegeneration. 


\subsection{Never-resting microglia in physiological conditions}

The original descriptions by Río-Hortega of microglia stained with his silver carbonate method revealed the morphological attributes of these cells, with their small soma and their long and ramified expansions [1]. Río-Hortega also noted that, although microglia was distributed throughout the brain, their density was higher in the gray compared to the white matter. He also proposed that microglial cells have a dynamic morphology, thereby introducing the notion that microglia adapt to the environment (see ref [2] for details). It is therefore surprising for decades after these pioneering studies microglial cells were considered being at rest in physiological conditions. The breakthrough occurred in 2005 when two groups imaged motility of microglia processes in the living brain of anesthetized transgenic mice expressing eGFP under control of microglia-specific promoter $[3,4]$. These studies revealed that microglia in physiological conditions constantly extend and retract their processes, leading to the notion that they permanently survey their environment. Subsequent imaging studies found that during this surveying activity, microglial cells establish contacts with synapses, while the frequency and duration of these contacts are modulated by activity and could also influence the fate and the activity of these synapses [5-12]. These pioneering studies not only demonstrated that microglial cells are never idle in physiological conditions but also that they are exeptionally sensitive to changes of their environment. Microglia ability to adapt to their local environment is nicely exemplified by the study of De Biase et al. who compared the phenotype of microglia in different nuclei of the basal ganglia and reported striking differences in terms of cell density and morphology, transcriptomic profiles and expression of potassium channels [13]. Remarkably, after depletion, repopulating microglia acquire the same phenotype characteristic of each area, strongly suggesting that local environmental cues, specific of each area, dictate the different microglia phenotypes. Other 
examples of the adaptability of microglia to local cues include the presence of a population of microglia in the neocortex closely associated with the axon initial segment of pyramidal cells [14], the specific properties of cerebellar microglia [15], the phagocytic activity of dentate gyrus microglia that regulates the fate of newborn neurons in the adult brain [16] and the large repertoire of phenotypes and functions expressed by microglia in different areas and at specific stages of the brain development [17].

Thus, microglial cells in physiological conditions are no longer considered as resting cells. These "never resting" or surveying immune cells dynamically interact with other components of the CNS parenchyma and these interactions determine their phenotype and thus their influence on CNS functions. In line with this new vision of microglia in physiological conditions, the notion of microglia activation in pathological conditions has dramatically evolved during the last decade.

\subsection{Multiple states of reactive microglia}

For decades microglial activation has been regarded as a stereotyped transformation of "resting" ramified microglia, in physiological conditions, into fully immunocompetent amoeboid macrophage upon rupture of CNS homeostasis or pathological stimulation. We know now that microglial activation is not an all-or-none process; it is progressive and at least partially reversible, it depends on the pathological context, the nature and the strength of the stimuli and on the settings in which these stimuli appear. Microglial responses to CNS disturbance involve two signaling principles: one is based on the appearance of factors that are usually absent, or at low concentration or in different configuration in physiological conditions (ON signals, e.g. microbial structures, cytokines, intracellular constituents released by damaged cells, protein aggregates, blood components); the second one is based on the disappearance of signals constitutively present in physiological conditions that contribute to 
maintaining the surveillance state of microglia (OFF signals, e.g. the chemokine fractalkine, the glycoproteins CD200 and SIRPa) $[18,19]$. Microglial cells are also endowed with a large repertoire of receptors for neurotransmitters and neuromodulators that can impact on their responses to pathological stimuli [20]. Thus, microglia in any given pathological context will be exposed to a specific set of $\mathrm{ON}$ and OFF signals and diffusible mediators that will determine a specific microglial phenotype. Different phenotypes mean different functions and indeed reactive microglia can express different important functional properties. For example, it was shown that differential stimulation of microglial cells by different cytokines or exogenous agents resulted in the acquisition of distinct phenotypes either offering neuroprotection or being cytotoxic [21].

Another example of the diversity of the triggers of microglial response is the expression of the purinergic receptor $\mathrm{P} 2 \mathrm{Y}_{12}$. This receptor is highly expressed by surveying microglial cells and regulates the extension of their processes toward extracellular nucleotides [22]. Downregulation of $\mathrm{P} 2 \mathrm{Y}_{12}$ protein and/or transcript is observed in several models of pathological conditions (e.g. in LPS-induced peripheral inflammation [22-24]; in amyotrophic lateral sclerosis (ALS) [25]; or in Alzheimer disease [26]) and has been considered as a landmark of microglia reactivity. Yet, in a mouse model of status epilepticus, we previously reported that the inflammatory reaction observed in the hippocampus was associated with a reactive phenotype of microglia characterized by morphological changes, proliferation, up-regulation of voltage-activated potassium channels and of purinergic signaling, including the upregulation of $\mathrm{P} 2 \mathrm{Y}_{12}$ functions [27].

Initial attempts to characterize microglia reactivity relied on low-throughput methodologies (e.g. morphological analysis, immunohistochemistry and electrophysiology). Although essential to infer the consequences for microglia cell biology, these approaches do not allow encompassing the diversity of responses mounted by microglial cells. With the medium- 
throughput molecular approaches becoming popular, which include the quantitative PCR (qPCR), it became possible to analyze simultaneously several tens of parameters/genes in a single cell population. These approaches help to reveal the existence of diverse microglial states (for review see [19]). Analogous to the Th1 and Th2 nomenclatures of T-lymphocytes [28] and in an attempt of classifying these reactive states, the concept of M1/M2 macrophage polarization was applied to microglia [29, 30]. Four main microglial states were defined: classically activated M1 microglia were proposed to have cytotoxic properties; M2a activated microglia showed an alternate activation phenotype and were supposedly involved in repair and regeneration; $\mathrm{M} 2 \mathrm{~b}$ microglia displayed an immunoregulatory phenotype; and M2c microglia had acquired-deactivating phenotype. It was further proposed that microglia can react along a spectrum of reactive states from which M1 and M2 were the extremes. One of the challenges beyond this concept was to identify, in different pathological conditions, the dominant microglia reaction state with the objective to infer the best tailored therapeutic strategy. In this regard, Chhor et al. [31] established the temporal expression of a battery of phenotypic markers in different in-vitro conditions of microglia polarization. As will be reviewed later, it has now been demonstrated that this concept is over-simplistic and doesn't consider that microglia are long lasting resident tissue macrophages highly adapted to the CNS environment [32]. Of note, the concept of M1/M2 macrophage polarization is also now being challenged [33-35].

It is now clear that there is not either a single or a discrete number of microglial reactive states but a diversity of phenotypes that are determined by a fine detection of environmental cues, which allows microglial cells to perform specific functions in different physiological and pathological conditions $[19,36,37]$. Although functional, morphological, immunohistochemical, and medium throughput analyses of microglia in pathological conditions initially pointed out to the existence of a diversity of reactive phenotypes $[19,36$, 
37], the actual phenotype diversity and exquisite plasticity of microglia have been further revealed by the advent of high throughput molecular approaches.

\subsection{Contribution of high-throughput approaches to the understanding of the diversity of microglial reaction}

Bulk RNA sequencing (RNA-Seq): At the beginning of the 2000's, the emergence of cellspecific transcriptome profiling approaches (mainly microarrays or RNA-seq based approaches) has empowered uncovering the role of microglia under both physiological and pathological conditions (for review see [38-41]. Based on high-throughput techniques, these approaches allowed to study the remodeling of the whole microglial transcriptome under pathological conditions. Of note, the expression profiling of microglia isolated from many different disease models failed to show evidence for microglia polarization along an M1-M2 axis, leading to the abandonment of this concept [42]. However, this does not mean that microglia reaction profiles cannot be categorized. Indeed, several kinetic analyses of the microglial response in a single disease have shown that microglia express specific patterns of response to neurodegenerative conditions [25, 43]. Additionally, comparison of microglial transcriptomic responses in different pathological conditions (including neurodegenerative and acute inflammatory conditions), led us to identify a core reaction signature [24]. Using more specific bioinformatic analyses such as co-variation studies, it is also possible to compare at the network level the remodeling of microglial transcriptomes in different pathological conditions. Hence, using the Weighted Gene co-expression Network Analysis (WGCNA) approach, Holtmann et al. [44] identified common transcriptional profiles for upregulated genes in the different neurodegenerative conditions and identified common traits to these disorders. 
Single-cell RNA-Seq (scRNA-Seq): Conventional RNA-seq approaches are based on transcriptomic analyses made on the scale of a whole cellular population. With the emergence of single cell high throughput approaches, another level of complexity of microglial reactivity can be investigated as it becomes possible to investigate the diversity of the microglial response at cellular resolution. The challenge is to identify sub-populations with specific functions having beneficial, neutral or deleterious effects on the disease progression. Using such approach, Keren-Shaul et al. [26] identified the Disease Associated Microglia (DAM) as a subpopulation of microglial cells in a mouse model of Alzheimer Disease (AD). In this model, DAM markers were mostly found in amyloid- $\beta$ plaque-associated microglia and relevance to the human pathology was demonstrated. Other studies identified DAM or DAMlike microglia in other neurodegenerative conditions including ALS, fronto-temporal dementia (FTD) and in an inducible mouse model of severe neurodegeneration [26, 45, 46]. Interestingly, applying trajectory inference methods to scRNA-seq data allows studying the transition of cells from one subtype to another. Thus, DAMs which relative abundance increases with disease progression, appear through a two-step activation process. First, homeostatic microglia transit to an intermediate (Stage 1 DAM) state in a TREM2independent manner, followed by a second TREM2-dependent transition to Stage 2 DAM [26]. As recently exemplified in a model of facial nerve axotomy, scRNA-seq also offers the possibility to study the functional relevance of transient microglial subpopulations over the time course of disease progression and recovery [47].

Epigenetic studies: Sequencing studies highlighted the extreme plasticity of microglia which finely tune their molecular identity in response to different stimuli. This coordinated regulation of gene expression requires changes in the composition and the structure of the chromatin. This is achieved through the action of epigenetic modulators, importance of which in controlling the microglial phenotypes is just starting being unraveled. Histone 
modifications, DNA methylation as well as microRNA expression have been shown to be involved in regulating microglial plasticity and their abilities to acquire specific phenotypes (reviewed in ref. [48]). For example, MafB, Mef2C and MeCP2 play key roles in controlling microglia reactivity upon challenges [49-51]. Thus, the diversity of the microglial reaction phenotypes arises both from their transcriptome and epigenome. Epigenetic mechanisms are likely to define the immune memory of microglia, a process that is thought to be involved in the differential responses of individuals to neurodegenerative conditions [52]. Involvement of epigenetics in regulating microglia reactivity raises important questions including: how long these long living cells are affected by earlier challenges and what can be the consequences in terms of progression of neurodegenerative disease conditions?

Proteomics: Although transcriptional profiling provides useful information regarding the functional status of a given cell population/subpopulation, genes are not the cell's effectors and discrepancies have been evidenced between gene and protein expression changes [53]. In this respect, proteomic studies are expected to provide an understanding of the pathological changes that is closer to the reality. Although recent advances have been made in mass spectrometry methods [54], quantitative proteomic studies of isolated microglia are still sparse [53, 55-57]. These studies highlighted modest overlap between expression observed at the transcriptomic and proteomic levels $[53,57]$, and emphasized the need to study microglial activation both at the transcript and protein levels to get precise insights into the molecular processes at work. Further improvement in the sensitivity of the technology will allow identifying more proteins. Analyses of isoforms and post-translational modifications of microglial proteins represent another major challenge toward the understanding of the functional role of microglia in health and disease.

\subsection{Microglial reaction signatures}


From many different studies of microglial reactions in different pathological contexts, it appears that microglial reasponse is a multifaceted process that is influenced by many parameters including but not limited to the cell's local environment and "history"; the nature, intensity and duration of the stimulus; the gender and the microbiome status; etc. Microglial reaction is also a highly dynamic process which further increases the diversity of phenotypes these cells can encompass.

Although highly complex, comparison of the changes observed in different experimental conditions identified common features of microglial reaction. By comparing the changes observed in five different experimental conditions, including acute neuroinflammation and neurodegenerative conditions, we defined a core microglial reaction signature (i.e. reactome; [24] corresponding to a set of 86 highly expressed genes that are deregulated in both inflammatory and neurodegenerative conditions. As expected, most of the reactome genes were also found in the DAM signature [26]. From these studies, it appears that microglia reaction is associated first with the loss of the homeostatic signature, characterized by the down-regulation of specific microglial genes including P2ry12, P2ry13, Slc2a5 and Sall1. Second, reactive microglia up-regulate genes involved in lysosomal and lipid metabolism pathways, and cytokine signaling, such as Ctss, Ctsz, Apoe and Il1ß (Fig. 1).

Besides this core reaction signature, comparison of deregulated gene lists as well as more refined meta-analyses studies show that microglia isolated in neurodegenerative conditions share consistent transcriptional profile whereas those isolated in acute inflammation conditions display a different signature [24, 25, 44].

The transcriptional microglial profiles observed in neurodegenerative conditions such as $A D$, FTD, ALS, Multiple Sclerosis (MS) and aging, correspond to that of the DAM, DAM-like and the microglial neurodegenerative phenotype (MGnD; [58], reviewed in ref. [59]). Recent studies based on brain injection of apoptotic neurons suggested that emergence of 
DAM/MGnD is consecutive to cell death [58] and relies on a battery of receptors, such as Trem2, Tyro3/Ax1/Mer (TAM) receptor kinases and P2ry12, which can recognize neurodegeneration-associated molecular patterns (NAMPs) and activate intracellular signaling cascades. The ApoE-Trem2 pathway appears to be key for the acquisition of DAM or DAM-like phenotypes [58]. Of note, because DAMs associate with cell death, they may represent subset of microglia that appear relatively late in neurodegenerative conditions, whereas more sparse or subtle phenotypes may appear sooner during disease progression.

Deregulated genes identified in microglia isolated from acute inflammation conditions have been shown to belong to a different gene network, significantly enriched for NF- $\mathrm{BB}$ signaling [44]. Using a scRNA-seq approach a recent study also confirmed that inflammatory associated microglia (IAM) exhibit a distinct transcriptional profile compared to DAM [60]. In addition, this study demonstrates that IAM is in fact a heterogeneous population, and identifies a subset of reactive microglia exhibiting a dimmer reactive state compared to the main population. Whether this subpopulation corresponds to cells that are less sensitive to inflammatory stimuli or cells that have recovered faster from a more pronounced reactive state is currently unknown.

Taken together, genome wide studies aimed at investigating in vivo reactive microglia have revealed common but also specific features to the reaction processes. Additional studies are needed to define disease- and stage-dependent microglial signatures. Heterogeneity in the reaction process also needs to be considered to provide a complete picture of microglial reaction. This will undoubtedly facilitate the design of more efficient therapeutic strategies.

\subsection{Potential confounding factors when studying microglia activation}

By nature, microglia are sensing cells whose phenotype changes depending on the signal they receive from their neighborhood. For this reason, experimenting with these cells is 
challenging as it is likely to influence their basic functions and impact the repertoire of genes they express.

Cell cultures: Microglial reaction was first studied using in vitro approaches either based on microglial cell lines or primary neonatal cultures [31]. Although these approaches proved to be useful and allowed to tackle the versatility of microglial cells, it is now clear that in vitro cultured microglial cells differs substantially from in vivo isolated microglia. Once in a petri dish, microglia exhibit an amoeboid morphology, proliferate [61, 62], quickly lose their homeostatic signature and start exhibiting a reactive profile $[63,64]$. Cultivating microglia in a serum free media, in presence of CSF1/IL-34, TGFß and cholesterol allows mimicking the morphology and dynamics of resting microglia, however it does not fully restore their homeostatic signature and microglia cultured under those conditions appear de-differentiated [63]. Co-culture of microglia with neurons also shifted the microglial signature towards a profile which is closer to that of homeostatic microglia thus further revealing the importance of brain derived signals to maintain microglia phenotype [65].

In vivo expriments: With the identification of specific extracellular microglia markers that can be used in flow cytometry [66] and the development of the CX3CR1 $1^{+/ G F P}$ mouse, in which microglia are the only brain cells to express eGFP [67], it became possible in the early 2000s, to isolate pure microglia cell population from different pathological conditions and to study the in vivo remodeling of microglia transcriptomes (reviewed [38-41]). Although, these approaches have been widely used and were proved useful for studying microglia reactions, it presents several limitations. Indeed, most current published protocols require the use of both mechanical and enzymatic dissociation, usually performed at $22^{\circ} \mathrm{C}$ or $37^{\circ} \mathrm{C}$, to obtain single cell suspension. Subsequent isolation steps often involve antibody binding which again might further impact on microglial transcriptome. Using a RiboTag approach, Haimon et al. [68] indeed demonstrated that classical microglia sorting protocols were tainted by artifacts 
introduced by tissue dissociation, cargo contamination and transcripts sequestered from ribosomes. Importantly, they demonstrate that these artifacts vary in control and pathological conditions. On the other hand, the RiboTag approach only allows detecting messengers that are being translated, thus giving access to the microglial translatome, which differs from the transcriptome. In addition to the RiboTag approach, new procedures such as the Act-Seq protocol [69] or the use of proteases operating at low temperature [70] open the way to perform cell dissociation with minimal transcriptomic perturbations but still need to be validated. These procedures might be useful to unravel the exact microglia transcriptome.

Human versus mouse microglia: Studying microglia reactivity provides a better understanding of the molecular processes involved in diseases initiation and progressions. The ultimate goal is to infer functions to reactive microglia, and in fine to design efficient therapeutic strategies with clinical benefit. The success of this workflow relies on the portability between results obtained in mice and in humans. Two simultaneous studies, using either a post-mortem [71] or surgical tissues [64] investigated gene expression profiles of human microglia and compared them to that of the mouse. Both highlighted that, overall, genes expressed in human and mouse microglia were very similar. Of interest to the microglia reaction issue, a good correlation between human and mouse microglia in response to neurodegeneration was observed $[26,44,58]$, thus validating the use of mouse models to study the roles of microglia in neuroinflammatory and neurodegenerative conditions. However, notwithstanding their global resemblance, a significant number of genes are differentially expressed in mouse and human microglia. Indeed, a number of immune genes are only present in human samples [64] and there are differences in the relative expression of lineage- and signal-dependent transcription factors in mice and humans [40]. Moreover, Galatro et al. [71] revealed that there is a limited overlap in age-related changes in human and mouse microglia, highlighting that data related to aged microglia in mouse must be interpreted and extrapolated to the 
human situation with caution. Translation of results from mice to humans is also hampered by the lack of tools to precisely characterize microglial reactivity in clinical studies: TSPO binding is so far the only way to study microglia reaction in a clinical context.

Development of cells reprogramming methods offers the possibility to generate human pluripotent cell lines (hiPSCs) from healthy individuals but also from patients with specific diseases. hiPSCs have recently been successfully differentiated into microglia $[65,72,73]$. Although obtaining microglia derived iPSCs with "normal" functions requires to grow them in presence of neurons (see [65]), such approaches represent powerful experimental models to decipher the roles of reactive microglia in human diseases.

\subsection{The yin and the yang of microglial reactivity}

Arising from in vitro studies which show that supernatant from LPS-activated microglia were toxic for the neurons, microglia reaction was initially considered as a negative event that needed to be contained. More recent data indicate that reactive microglia can have positive impact on the development of CNS pathologies. Indeed, reactive microglia can release antiinflammatory mediators such as IL-10 or TGF- $\beta$ which may positively impact on the disease resolution. In stroke models, reactive microglia are generally considered to play deleterious roles, however increasing evidence showed that microglial reactivity could have beneficial effects through reduction of the lesions size and the neuronal death (reviewed in [74]).

Whether DAM/MGnD microglia are beneficial or deleterious for progression of neurodegenerative diseases remains unclear. Several genes deregulated upon transition from homeostatic to DAM phenotype have been associated with risk factors in neurodegenerative conditions, especially in AD. As a whole, functional studies based on risk genes bearing mutations found in $\mathrm{AD}$ patients point towards a protective role for DAM in AD and suggest that unleashing microglia checkpoints in the early stages of the diseases may be beneficial 
[75]. However, some other studies suggest that the outcome of DAM presence may depend on the stage of the disease [76].

As resolution in microglia reaction's analysis progresses, it becomes clear that the diversity of the microglial response to injury is much greater than initially anticipated. It seems now likely that multiple microglial subpopulations with differential roles in disease progression coexist in the same environment. Further investigations are required to precisely characterize the microglial diversity at critical disease's stages and to identify the specific functions associated with the different subpopulations in order to infer their functional roles. Such heterogeneity within the microglia cell population represents both a challenge and an opportunity: the existence of distinctive subpopulations indeed supports the design of specific treatments targeting specific subpopulations with the aims of either promoting the beneficial subpopulations and/or hampering the deleterious ones.

\subsection{Concluding remarks}

From the last twenty years, we learned that as the main immune cells of the CNS, microglia play the role of an orchestra conductor for the maintenance of the brain's homeostasis. This role is made possible through the high plasticity / reactivity of this cell type.

"Microglial reaction" thus appears as an umbrella term used to describe a great variety of functional and morphological responses toward different triggers, including transient homeostatic imbalance, inflammation, chronic neurodegenerative conditions or environmental factors. Microglia reactivity is driven by two main signaling principles: responses caused by the acquisition of receptor signaling and responses caused by disrupted signaling. The ultimate goal for microglia is to eliminate or isolate the threat and restore homeostasis. From an all-or-none process, our understanding of the microglial reaction has evolved towards a "kaleidoscope" vision [38]. Yet, some microglial subpopulations, such as the "dark 
microglia" may be understudied because they are not easily detected [77]. Interestingly, recent studies have highlighted that microglia reaction is finely tuned through several mechanisms, referred as microglial checkpoints, that restrain microglia immune activation and promotes homeostatic functions [75].

A great part of our understanding of microglia reactivity's diversity arose from the development of technological breakthroughs such as live imaging and high-throughput sequencing (RNAseq, ScRNAseq, ChipSeq, etc). Further breakthroughs, including the development of sensitive proteomic approaches, will certainly push forward of understanding of the microglial biology in health and disease. Integration of the complex and multifactorial regulation of microglial reaction states (at the epigenetic, genetic and protein levels) in disease models will be required to design safe and efficient therapeutic strategies for diseases in which microglia play a major role.

\section{Acknowledgements}

Work in Audinat lab is funded by grants from the Fondation pour la Recherche Médicale (FRM: DEQ20140329488) and the European Commission (ERA-NET Neuron BrIE; ECH2020 MSCA-ITN EU-GliaPhD No. 722053). Work in Rassendren's lab is funded by grants from the Fondation Plan Alzheimer (MicroMad).

\section{Figure legends:}

\section{Figure 1: Common microglial reactive signature}

Venn diagram showing the overlap of the different microglia reaction signatures in (i) DAMs

(purple), (ii) MGnD (red), (iii) IAMs (green) and (iv) Reactome (yellow). The list of genes associated with the different sub-groups is available in supplementary Table 1. 
Note that the gene list sizes for the different reaction signatures (Fig. 1) is highly dependent on the way the authors define the microglial reaction signature. Indeed, DAMs list is a set of 1037 coding genes identified through scRNA-seq as being significantly deregulated (False Discovery Rate (FDR) <0.05) between DAMs and homeostatic microglia clusters [26]; MGnD genes are a set of 95 genes, identified through k-means clustering of 550 preselected genes, that significantly affected $\mathrm{Fc}$ receptor-like $\mathrm{S}$ positive (FCRLS+) microglia during aging and disease [58]; IAMs list is a set of 2261 coding genes identified through scRNA-seq as being significantly deregulated $($ FDR $<0.05)$ between LPS-treated and homeostatic microglia clusters [60]; the Reactome list corresponds to a set of 86 highly expressed genes (selected from highly expressed and deregulated genes under inflammatory conditions) that are deregulated in both inflammatory and neurodegenerative conditions [24].

\section{References}

1. Sierra A, de Castro F, Del Rio-Hortega J, et al. (2016) The "Big-Bang" for modern glial biology: Translation and comments on Pio del Rio-Hortega 1919 series of papers on microglia. Glia. 64(11): p. 1801-1840.

2. Tremblay ME, Lecours C, Samson L, et al. (2015) From the Cajal alumni Achucarro and Rio-Hortega to the rediscovery of never-resting microglia. Front Neuroanat. 9: p. 45.

3. Davalos D, Grutzendler J, Yang G, et al. (2005) ATP mediates rapid microglial response to local brain injury in vivo. Nat Neurosci. 8(6): p. 752-758.

4. Nimmerjahn A, Kirchhoff F, and Helmchen $F(2005)$ Resting microglial cells are highly dynamic surveillants of brain parenchyma in vivo. Science. 308(5726): p. 1314-1318.

5. Dissing-Olesen L, LeDue JM, Rungta RL, et al. (2014) Activation of neuronal NMDA receptors triggers transient ATP-mediated microglial process outgrowth. J Neurosci. 34(32): p. 10511-10527.

6. Eyo UB and $\mathrm{Wu} \sqcup$ (2013) Bidirectional microglia-neuron communication in the healthy brain. Neural Plast. 2013: p. 456857.

7. Li Y, Du XF, Liu CS, et al. (2012) Reciprocal Regulation between Resting Microglial Dynamics and Neuronal Activity In Vivo. Dev Cell. 23(6): p. 1189-1202.

8. Parkhurst CN, Yang G, Ninan I, et al. (2013) Microglia Promote Learning-Dependent Synapse Formation through Brain-Derived Neurotrophic Factor. Cell. 155(7): p. 15961609.

9. Pfeiffer T, Avignone E, and Nagerl UV (2016) Induction of hippocampal long-term potentiation increases the morphological dynamics of microglial processes and prolongs their contacts with dendritic spines. Sci Rep. 6: p. 32422. 
10. Sipe GO, Lowery RL, Tremblay ME, et al. (2016) Microglial P2Y12 is necessary for synaptic plasticity in mouse visual cortex. Nat Commun. 7: p. 10905.

11. Tremblay ME and Majewska AK (2011) A role for microglia in synaptic plasticity? Commun Integr Biol. 4(2): p. 220-222.

12. Wake H, Moorhouse AJ, Jinno S, et al. (2009) Resting microglia directly monitor the functional state of synapses in vivo and determine the fate of ischemic terminals. $J$ Neurosci. 29(13): p. 3974-3980.

13. De Biase LM, Schuebel KE, Fusfeld ZH, et al. (2017) Local Cues Establish and Maintain Region-Specific Phenotypes of Basal Ganglia Microglia. Neuron. 95(2): p. 341-356 e6.

14. Baalman K, Marin MA, Ho TS, et al. (2015) Axon initial segment-associated microglia. J Neurosci. 35(5): p. 2283-2292.

15. Stowell RD, Wong EL, Batchelor HN, et al. (2018) Cerebellar microglia are dynamically unique and survey Purkinje neurons in vivo. Dev Neurobiol. 78(6): p. 627-644.

16. Sierra A, Encinas JM, Deudero JJ, et al. (2010) Microglia shape adult hippocampal neurogenesis through apoptosis-coupled phagocytosis. Cell Stem Cell. 7(4): p. 483495.

17. Mosser CA, Baptista S, Arnoux I, et al. (2017) Microglia in CNS development: Shaping the brain for the future. Prog Neurobiol. 149-150: p. 1-20.

18. Biber K, Neumann H, Inoue K, et al. (2007) Neuronal 'On' and 'Off' signals control microglia. Trends Neurosci. 30(11): p. 596-602.

19. Hanisch UK and Kettenmann H (2007) Microglia: active sensor and versatile effector cells in the normal and pathologic brain. Nat Neurosci. 10(11): p. 1387-1394.

20. Kettenmann H, Hanisch UK, Noda M, et al. (2011) Physiology of microglia. Physiol Rev. 91(2): p. 461-553.

21. Butovsky O, Talpalar AE, Ben-Yaakov K, et al. (2005) Activation of microglia by aggregated beta-amyloid or lipopolysaccharide impairs MHC-II expression and renders them cytotoxic whereas IFN-gamma and IL-4 render them protective. Mol Cell Neurosci. 29(3): p. 381-393.

22. Haynes SE, Hollopeter G, Yang G, et al. (2006) The P2Y12 receptor regulates microglial activation by extracellular nucleotides. Nat Neurosci. 9(12): p. 1512-1519.

23. Bennett ML, Bennett FC, Liddelow SA, et al. (2016) New tools for studying microglia in the mouse and human CNS. Proc Natl Acad Sci U S A. 113(12): p. E1738-46.

24. Hirbec H, Marmai C, Duroux-Richard I, et al. (2018) The microglial reaction signature revealed by RNAseq from individual mice. Glia. 66(5): p. 971-986.

25. Chiu IM, Morimoto ET, Goodarzi H, et al. (2013) A neurodegeneration-specific geneexpression signature of acutely isolated microglia from an amyotrophic lateral sclerosis mouse model. Cell Rep. 4(2): p. 385-401.

26. Keren-Shaul H, Spinrad A, Weiner A, et al. (2017) A Unique Microglia Type Associated with Restricting Development of Alzheimer's Disease. Cell. 169(7): p. 1276-1290 e17.

27. Avignone E, Ulmann L, Levavasseur F, et al. (2008) Status epilepticus induces a particular microglial activation state characterized by enhanced purinergic signaling. $J$ Neurosci. 28(37): p. 9133-9144.

28. Mills CD, Kincaid K, Alt JM, et al. (2000) M-1/M-2 macrophages and the Th1/Th2 paradigm. J Immunol. 164(12): p. 6166-6173.

29. Kreider T, Anthony RM, Urban JF, Jr., et al. (2007) Alternatively activated macrophages in helminth infections. Curr Opin Immunol. 19(4): p. 448-453. 
30. Martinez FO, Sica A, Mantovani A, et al. (2008) Macrophage activation and polarization. Front Biosci. 13: p. 453-461.

31. Chhor V, Le Charpentier T, Lebon S, et al. (2013) Characterization of phenotype markers and neuronotoxic potential of polarised primary microglia in vitro. Brain Behav Immun. 32: p. 70-85.

32. Ransohoff RM (2016) A polarizing question: do M1 and M2 microglia exist? Nat Neurosci. 19(8): p. 987-991.

33. Ginhoux F, Schultze JL, Murray PJ, et al. (2016) New insights into the multidimensional concept of macrophage ontogeny, activation and function. Nat Immunol. 17(1): p. 34-40.

34. Murray PJ, Allen JE, Biswas SK, et al. (2014) Macrophage activation and polarization: nomenclature and experimental guidelines. Immunity. 41(1): p. 14-20.

35. Xue J, Schmidt SV, Sander J, et al. (2014) Transcriptome-based network analysis reveals a spectrum model of human macrophage activation. Immunity. 40(2): p. 274288.

36. Kierdorf $\mathrm{K}$ and Prinz M (2013) Factors regulating microglia activation. Front Cell Neurosci. 7: p. 44.

37. Ransohoff RM and Perry VH (2009) Microglial physiology: unique stimuli, specialized responses. Annu Rev Immunol. 27: p. 119-145.

38. Dubbelaar ML, Kracht L, Eggen BJL, et al. (2018) The Kaleidoscope of Microglial Phenotypes. Front Immunol. 9: p. 1753.

39. Hirbec HE, Noristani HN, and Perrin FE (2017) Microglia Responses in Acute and Chronic Neurological Diseases: What Microglia-Specific Transcriptomic Studies Taught (and did Not Teach) Us. Front Aging Neurosci. 9: p. 227.

40. Holtman IR, Skola D, and Glass CK (2017) Transcriptional control of microglia phenotypes in health and disease. J Clin Invest. 127(9): p. 3220-3229.

41. Sousa C, Biber K, and Michelucci A (2017) Cellular and Molecular Characterization of Microglia: A Unique Immune Cell Population. Front Immunol. 8: p. 198.

42. Crotti A and Ransohoff RM (2016) Microglial Physiology and Pathophysiology: Insights from Genome-wide Transcriptional Profiling. Immunity. 44(3): p. 505-515.

43. Noristani HN, Gerber YN, Sabourin JC, et al. (2017) RNA-Seq Analysis of Microglia Reveals Time-Dependent Activation of Specific Genetic Programs following Spinal Cord Injury. Front Mol Neurosci. 10: p. 90.

44. Holtman IR, Raj DD, Miller JA, et al. (2015) Induction of a common microglia gene expression signature by aging and neurodegenerative conditions: a co-expression meta-analysis. Acta Neuropathol Commun. 3: p. 31.

45. Friedman BA, Srinivasan K, Ayalon G, et al. (2018) Diverse Brain Myeloid Expression Profiles Reveal Distinct Microglial Activation States and Aspects of Alzheimer's Disease Not Evident in Mouse Models. Cell Rep. 22(3): p. 832-847.

46. Mathys H, Adaikkan C, Gao F, et al. (2017) Temporal Tracking of Microglia Activation in Neurodegeneration at Single-Cell Resolution. Cell Rep. 21(2): p. 366-380.

47. Tay TL, Sagar, Dautzenberg J, et al. (2018) Unique microglia recovery population revealed by single-cell RNAseq following neurodegeneration. Acta Neuropathol Commun. 6(1): p. 87.

48. Cheray M and Joseph B (2018) Epigenetics Control Microglia Plasticity. Front Cell Neurosci. 12: p. 243. 
49. Cronk JC, Derecki NC, Ji E, et al. (2015) Methyl-CpG Binding Protein 2 Regulates Microglia and Macrophage Gene Expression in Response to Inflammatory Stimuli. Immunity. 42(4): p. 679-691.

50. Deczkowska A, Matcovitch-Natan O, Tsitsou-Kampeli A, et al. (2017) Mef2C restrains microglial inflammatory response and is lost in brain ageing in an IFN-I-dependent manner. Nat Commun. 8(1): p. 717.

51. Matcovitch-Natan O, Winter DR, Giladi A, et al. (2016) Microglia development follows a stepwise program to regulate brain homeostasis. Science. 353(6301): p. aad8670.

52. Wendeln AC, Degenhardt K, Kaurani L, et al. (2018) Innate immune memory in the brain shapes neurological disease hallmarks. Nature. 556(7701): p. 332-338.

53. Sharma K, Schmitt S, Bergner CG, et al. (2015) Cell type- and brain region-resolved mouse brain proteome. Nat Neurosci. 18(12): p. 1819-1831.

54. Umoh ME, Dammer EB, Dai J, et al. (2018) A proteomic network approach across the ALS-FTD disease spectrum resolves clinical phenotypes and genetic vulnerability in human brain. EMBO Mol Med. 10(1): p. 48-62.

55. Boza-Serrano A, Yang Y, Paulus A, et al. (2018) Innate immune alterations are elicited in microglial cells before plaque deposition in the Alzheimer's disease mouse model 5xFAD. Sci Rep. 8(1): p. 1550.

56. Flowers A, Bell-Temin H, Jalloh A, et al. (2017) Proteomic anaysis of aged microglia: shifts in transcription, bioenergetics, and nutrient response. J Neuroinflammation. 14(1): p. 96.

57. Rangaraju S, Dammer EB, Raza SA, et al. (2018) Quantitative proteomics of acutelyisolated mouse microglia identifies novel immune Alzheimer's disease-related proteins. Mol Neurodegener. 13(1): p. 34.

58. Krasemann S, Madore C, Cialic R, et al. (2017) The TREM2-APOE Pathway Drives the Transcriptional Phenotype of Dysfunctional Microglia in Neurodegenerative Diseases. Immunity. 47(3): p. 566-581 e9.

59. Deczkowska A, Keren-Shaul H, Weiner A, et al. (2018) Disease-Associated Microglia: A Universal Immune Sensor of Neurodegeneration. Cell. 173(5): p. 1073-1081.

60. Sousa C, Golebiewska A, Poovathingal SK, et al. (2018) Single-cell transcriptomics reveals distinct inflammation-induced microglia signatures. EMBO Rep. 19(11).

61. Stansley B, Post J, and Hensley K (2012) A comparative review of cell culture systems for the study of microglial biology in Alzheimer's disease. J Neuroinflammation. 9: $p$. 115.

62. Witting A and Moller T (2011) Microglia cell culture: a primer for the novice. Methods Mol Biol. 758: p. 49-66.

63. Bohlen CJ, Bennett FC, Tucker AF, et al. (2017) Diverse Requirements for Microglial Survival, Specification, and Function Revealed by Defined-Medium Cultures. Neuron. 94(4): p. 759-773 e8.

64. Gosselin D, Skola D, Coufal NG, et al. (2017) An environment-dependent transcriptional network specifies human microglia identity. Science. 356(6344).

65. Takata K, Kozaki T, Lee CZW, et al. (2017) Induced-Pluripotent-Stem-Cell-Derived Primitive Macrophages Provide a Platform for Modeling Tissue-Resident Macrophage Differentiation and Function. Immunity. 47(1): p. 183-198 e6.

66. de Haas $\mathrm{AH}$, Boddeke HW, Brouwer N, et al. (2007) Optimized isolation enables ex vivo analysis of microglia from various central nervous system regions. Glia. 55(13): $p$. 1374-1384. 
67. Jung S, Aliberti J, Graemmel P, et al. (2000) Analysis of fractalkine receptor CX(3)CR1 function by targeted deletion and green fluorescent protein reporter gene insertion. Mol Cell Biol. 20(11): p. 4106-4114.

68. Haimon Z, Volaski A, Orthgiess J, et al. (2018) Re-evaluating microglia expression profiles using RiboTag and cell isolation strategies. Nat Immunol. 19(6): p. 636-644.

69. Wu YE, Pan L, Zuo Y, et al. (2017) Detecting Activated Cell Populations Using SingleCell RNA-Seq. Neuron. 96(2): p. 313-329 e6.

70. Adam M, Potter AS, and Potter SS (2017) Psychrophilic proteases dramatically reduce single-cell RNA-seq artifacts: a molecular atlas of kidney development. Development. 144(19): p. 3625-3632.

71. Galatro TF, Holtman IR, Lerario AM, et al. (2017) Transcriptomic analysis of purified human cortical microglia reveals age-associated changes. Nat Neurosci. 20(8): p. 1162-1171.

72. Douvaras P, Sun B, Wang M, et al. (2017) Directed Differentiation of Human Pluripotent Stem Cells to Microglia. Stem Cell Reports. 8(6): p. 1516-1524.

73. Muffat J, Li Y, Yuan B, et al. (2016) Efficient derivation of microglia-like cells from human pluripotent stem cells. Nat Med. 22(11): p. 1358-1367.

74. Ma Y, Wang J, Wang Y, et al. (2017) The biphasic function of microglia in ischemic stroke. Prog Neurobiol. 157: p. 247-272.

75. Deczkowska A, Amit I, and Schwartz M (2018) Microglial immune checkpoint mechanisms. Nat Neurosci. 21(6): p. 779-786.

76. Jay TR, Hirsch AM, Broihier ML, et al. (2017) Disease Progression-Dependent Effects of TREM2 Deficiency in a Mouse Model of Alzheimer's Disease. J Neurosci. 37(3): p. 637-647.

77. Bisht K, Sharma KP, Lecours C, et al. (2016) Dark microglia: A new phenotype predominantly associated with pathological states. Glia. 64(5): p. 826-839. 